\title{
Researching on Hawking Effect in a Kerr Space Time via Open Quantum System Approach
}

\author{
Xian-Ming Liu ${ }^{1}$ and Wen-Biao Liu ${ }^{2}$ \\ ${ }^{1}$ Department of Physics, Hubei University for Nationalities, Enshi Hube 445000, China \\ ${ }^{2}$ Department of Physics, Institute of Theoretical Physics, Beijing Normal University, Beijing 100875, China \\ Correspondence should be addressed to Xian-Ming Liu; xianmingliu@mail.bnu.edu.cn
}

Received 21 January 2014; Accepted 30 January 2014; Published 9 March 2014

Academic Editor: Xiaoxiong Zeng

Copyright (c) 2014 X.-M. Liu and W.-B. Liu. This is an open access article distributed under the Creative Commons Attribution License, which permits unrestricted use, distribution, and reproduction in any medium, provided the original work is properly cited. The publication of this article was funded by $\mathrm{SCOAP}^{3}$.

\begin{abstract}
It has been proposed that Hawking radiation from a Schwarzschild or a de Sitter spacetime can be understood as the manifestation of thermalization phenomena in the framework of an open quantum system. Through examining the time evolution of a detector interacting with vacuum massless scalar fields, it is found that the detector would spontaneously excite with a probability the same as the thermal radiation at Hawking temperature. Following the proposals, the Hawking effect in a Kerr space time is investigated in the framework of an open quantum systems. It is shown that Hawking effect of the Kerr space time can also be understood as the the manifestation of thermalization phenomena via open quantum system approach. Furthermore, it is found that near horizon local conformal symmetry plays the key role in the quantum effect of the Kerr space time.
\end{abstract}

\section{Introduction}

Hawking radiation arising from the quantization of matter field in a curved background space-time with the event horizon is a prominent quantum effect. The research to understand Hawking radiation, which is related to general relativity, quantum theory, and thermodynamics, has attracted widespread interest in the physics community. Since Hawking's original derivation of black hole thermal radiation [1-3], several alternative methods have been proposed, such as Damour-Ruffini method $[4,5]$, the tunneling method [6$11]$, and gravitational anomaly method $[12,13]$.

However, from a physical viewpoint, the black hole thermodynamics system should be more like a nonequilibrium system rather than an equilibrium system. Hawking effect should be investigated in the frame of nonequilibrium statistics physics. In quantum mechanics and nonequilibrium statistics physics, the open quantum theory system has gotten a lot of successful development [14]. The quantum dynamics of an open quantum system characterized by the effects of decoherence and dissipation cannot be represented in terms of a unitary time evolution. It has been applied to quantum information science, modern quantum optics, atomic and many-body systems, soft condensed matter physics, and biophysics. Recently, in the paradigm of open quantum system, based on [15], Yu and Zhang proposed a new insight to understand Hawking radiation in a Schwarzschild space time [16]. Through examining the time evolution of a detector interacting with vacuum massless scalar field, they got a conclusion that the detector in both Unruh and HartleHawking vacua would spontaneously excite with a nonvanishing probability the same as Hawking thermal radiation from the black hole. This new approach has been extended to understand the Gibbons-Hawking effect of de Sitter spacetime [17]. However, there remain some challenges to study Hawking radiation from a generic Kerr space time under the manifestation of thermalization phenomena in an open quantum system.

Our motivation comes from the fact that the near-horizon geometry plays the key role to the character of a black hole space time [18-29]. In 1998, Strominger [18] discussed the near-horizon asymptotic symmetry in a Kerr black hole and found that there was a holographic duality between extremal and near-extremal Kerr black hole and a 2-dimensional 
conformal field theory. In $[23,24]$, it was shown that around the horizon of a Kerr space time, the scalar field theory can be reduced to a 2-dimensional effective field theory. Now, the thermal radiation of scalar particles from a Kerr black hole can be derived on the basis of a conformal symmetry arising from the near-horizon geometry [29].

Using the near-horizon geometry and open quantum system approach, Hawking effect in a Kerr space time will be investigated. We will examine the time evolution of a static detector (modeled by a two-level atom) outside a Kerr space time immersed in a vacuum massless scalar field. The dynamics of the detector can be obtained from the complete time evolution describing the total system (detector plus external field) by integrating over the field degrees of freedom. Our results show that the detector would spontaneously excite in the Unruh vacuum state with a probability the same as the thermal radiation at Hawking temperature, indicating that Hawking radiation from a Kerr space time can be understood as the manifestation of thermalization phenomena in the framework of open quantum systems. The conformal invariance of the wave equation near the horizon is at the key point of Hawking quantum effect in the Kerr space time.

The organization of our paper is as follows. In Section 2, we will review the basic formulae, including the master equation describing the system of the detector plus external vacuum scalar field in the weak-coupling limit and the reduced dynamical equation for the finite time evolution of the detector. In Section 3, the dimensional reduction technique is used to investigate the massless scalar field in a Kerr space time, and the Wightman function is obtained. In Section 4, applying the method and results of the preceding sections to calculate the probability of a spontaneous transition of the detector from the ground state to the excited state outside a Kerr space time. Finally, some discussions and conclusions will be given in Section 5 .

\section{Review of the Open Quantum System Approach}

In this section, we will review the open quantum system approach to get the master equation describing the combined system $B+S$, where a static detector (two-level atom) as an open system $S$ which is coupled to another quantum system $B$ of a vacuum massless scalar field in a Kerr space time. Our derivation mostly follows the works in [15-17]. Here, we will consider the evolution of the static detector in the proper time and assume the combined system $(B+S)$ to be initially prepared in a factorized state, with the detector keeping static in the exterior region of the Kerr black hole and the field keeping in vacuum state. The static detector is a two-level simplest quantum system whose Hilbert space is spanned over just two states, an excited state $|+\rangle$, and a ground state $|-\rangle$. The Hilbert space of such a system is equivalent to that of a spin-(1/2) system. So the states of the detector can be represented by a $2 \times 2$ density matrix, which is Hermitian $\rho^{\dagger}=\rho$, and normalized $\operatorname{Tr}(\rho)=1$ with $\operatorname{det}(\rho) \geqslant 0$. For simplicity, the Hamiltonian of the detector $H_{S}$ may be taken as

$$
H_{S}=\frac{\omega_{0}}{2} \sigma_{3}
$$

where $\sigma_{3}$ is the Pauli matrix and $\omega_{0}$ is the energy level spacing. The standard Hamiltonian of massless, free scalar field in a Kerr space time can be denoted as $H_{B}$, which will be discussed in detail in Section 3. The interaction Hamiltonian of the detector with the scalar field can be denoted as

$$
H_{I}^{\prime}=\sigma_{3} \phi(x) \text {. }
$$

Therefore, the Hilbert space of the total system $S+B$ is given by the tensor product space $\mathscr{H}=\mathscr{H}_{S} \otimes \mathscr{H}_{B}$. The total Hamiltonian $H(t)$ can be taken as

$$
H=H_{S} \otimes I_{B}+I_{S} \otimes H_{B}+\lambda H_{I}^{\prime},
$$

where $\lambda$ is the coupling constant and, $I_{S}$ and $I_{B}$ denote the identity operators in $\mathscr{H}_{S}$ and $\mathscr{H}_{B}$, respectively.

Now in order to get the reduced dynamics of the subsystem $S$, we assume that the interaction between the detector and the scalar field is weak as $\lambda$ is small and the finite time evolution describing the dynamics of the detector takes the form of a one-parameter semigroup of completely positive map.

Initially, the complete system is described by the total density matrix $\rho_{\text {tot }}=\rho(0) \otimes|0\rangle\langle 0|$, where $\rho(0)$ is the initial reduced density matrix of the detector and $|0\rangle$ is the Kerr space-time vacuum state of field $\phi(x)$. In the frame of the atom, the evolution in the proper time $\tau$ of the total density $\rho_{\text {tot }}$ of the complete system satisfies

$$
\frac{\partial \rho_{\mathrm{tot}}(\tau)}{\partial \tau}=-i L_{H}\left[\rho_{\mathrm{tot}}(\tau)\right]
$$

which is often referred to the von Neumann or Liouvillevon Neumann equation, where $L_{H}$ represents the Liouville operator associated with $H$ as follows:

$$
L_{H}[S] \equiv[H, S] .
$$

The dynamics of the detector can be obtained by summing over the degrees of freedom of the field $\phi$; that is, by applying to $\rho_{\text {tot }}(\tau)$ with the trace projection operator $P$ as follows:

$$
\rho(\tau)=P\left[\rho_{\text {tot }}(\tau)\right] \equiv \operatorname{Tr}_{\phi}\left[\rho_{\text {tot }}(\tau)\right]
$$

In the limit of weak coupling, we can find that the reduced density obeys an equation in the Kossakowski-Lindblad form [30-32] as follows:

$$
\frac{\partial \rho(\tau)}{\partial \tau}=-i\left[H_{\mathrm{eff}}, \rho(\tau)\right]+\mathscr{L}[\rho(\tau)]
$$

where

$$
\mathscr{L}[\rho]=\frac{1}{2} \sum_{i, j=1}^{3} a_{i j}\left[2 \sigma_{j} \rho \sigma_{i}-\sigma_{i} \sigma_{j} \rho-\rho \sigma_{i} \sigma_{j}\right] .
$$


The matrix $a_{i j}$ and the effective Hamiltonian $H_{\text {eff }}$ are determined by the Fourier transform $\mathscr{G}(\lambda)$ and Hilbert transform $\mathscr{K}(\lambda)$ of the vacuum field correlation function (the Wightman function) as follows:

$$
G^{+}(x-y)=\langle 0|\phi(x) \phi(y)| 0\rangle,
$$

and they are defined as

$$
\begin{gathered}
\mathscr{G}(\lambda)=\int d \tau e^{i \lambda \tau} G^{+}(x(\tau)), \\
\mathscr{K}(\lambda)=\frac{P}{\pi i} \int d \omega \frac{\mathscr{G}(\omega)}{\omega-\lambda} .
\end{gathered}
$$

The coefficients of the Kossakowski matrix $a_{i j}$ can be written as

$$
a_{i j}=A \delta_{i j}-i B \epsilon_{i j k} \delta_{k 3}+C \delta_{i 3} \delta_{j 3}
$$

with

$$
\begin{gathered}
A=\frac{1}{2}\left[\mathscr{G}\left(\omega_{0}\right)+\mathscr{G}\left(-\omega_{0}\right)\right], \\
B=\frac{1}{2}\left[\mathscr{G}\left(\omega_{0}\right)-\mathscr{G}\left(-\omega_{0}\right)\right], \\
C=\mathscr{G}(0)-A .
\end{gathered}
$$

The effective Hamiltonian $H_{\text {eff }}$ contains a correction term, the so-called Lamb shift, and one can find that it can be obtained by replacing $\omega_{0}$ in $H_{s}$ with a renormalized energy level spacing $\Omega$ as follows:

$$
H_{\mathrm{eff}}=\frac{\Omega}{2} \sigma_{3}=\omega_{0}+i\left[\mathscr{K}\left(-\omega_{0}\right)-\mathscr{K}\left(\omega_{0}\right)\right] \sigma_{3},
$$

where a suitable subtraction is assumed in the definition of $\mathscr{K}\left(-\omega_{0}\right)-\mathscr{K}\left(\omega_{0}\right)$ to remove the logarithmic divergence which would otherwise be presented.

To facilitate the discussion of the properties of solutions for (7) and (8), let us express the density matrix in terms of the Pauli matrices as follows:

$$
\rho(\tau)=\frac{1}{2}\left(1+\sum_{i=1}^{3} \rho_{i}(\tau) \sigma_{i}\right) .
$$

Substituting (14) into (8), the Bloch vector $|\rho(\tau)\rangle$ of components $\rho_{1}(\tau), \rho_{2}(\tau), \rho_{3}(\tau)$ satisfies

$$
\frac{\partial}{\partial \tau}|\rho(\tau)\rangle=-2 \mathscr{H}|\rho(\tau)\rangle+|\eta\rangle,
$$

where $|\eta\rangle$ denotes a constant vector $\{0,0,-4 B\}$. The exact form of the matrix $\mathscr{H}$ reads

$$
\mathscr{H}=\left(\begin{array}{ccc}
2 A+C & \frac{\Omega}{2} & 0 \\
-\frac{2}{\Omega} & 2 A+C & 0 \\
0 & 0 & A
\end{array}\right) .
$$

Equation (15) can be solved exactly and its solution is

$$
|\rho(\tau)\rangle=e^{-2 \mathscr{H} \tau}|\rho(0)\rangle+\left(1-e^{-2 \mathscr{H} \tau}\right)\left|\rho_{\infty}\right\rangle,
$$

where

$$
\left|\rho_{\infty}\right\rangle=\frac{1}{2} \mathscr{H}^{-1}|\eta\rangle=-\frac{B}{A}\left(\begin{array}{l}
0 \\
0 \\
1
\end{array}\right),
$$

the matrix $e^{-2 \mathscr{H} \tau}$ is defined by series expansion as usual. However, $\mathscr{H}$ obeys a cubic eigenvalue equation, so powers of $\mathscr{H}$ higher than 2 can always be written in terms of combinations of $\mathscr{H}^{2}, \mathscr{H}$, and $I$. Actually, three eigenvalues of $\mathscr{H}$ are $\lambda_{1}=$ $2 A, \lambda_{ \pm}=(2 A+C) \pm i \Omega / 2$. We can write

$$
\begin{aligned}
e^{-2 \mathscr{H} \tau}=\frac{4}{\Omega^{2}+4 C^{2}}\left\{e^{-2 A \tau} \Lambda_{1}+2 e^{-2(2 A+C) \tau}\right. & \\
& \left.\times\left[\Lambda_{2} \cos (\Omega \tau)+\Lambda_{3} \frac{\sin (\Omega \tau)}{\Omega}\right]\right\},
\end{aligned}
$$

where

$$
\begin{aligned}
\Lambda_{1}= & {\left[(2 A+C)^{2}+\frac{(\Omega)^{2}}{4}\right] I-2(2 A+C) \mathscr{H}+\mathscr{H}^{2}, } \\
\Lambda_{2}= & -2 A(A+C) I+(2 A+C) \mathscr{H}-\frac{1}{2} \mathscr{H}^{2}, \\
\Lambda_{3}= & 2 A\left[\frac{\Omega^{2}}{4}-C(2 A+C)\right] I \\
& +\left[C(4 A+C)-\frac{\Omega^{2}}{4}\right] \mathscr{H}-C \mathscr{H}^{2} .
\end{aligned}
$$

Equation (19) reveals that a freely falling atom in a Kerr space time is subjected to the effects of decoherence and dissipation by the exponentially decaying factors including the real parts of the eigenvalues of $\mathscr{H}$ and oscillating terms associated with the imaginary part. These nonunitary effects can be analyzed by examining the evolution behavior in time of suitable atom observable. For any observable of the atom represented by a Hermitian operator $\mathcal{O}$, the behavior of its mean value is determined by

$$
\langle\mathcal{O}\rangle=\operatorname{Tr}[\mathcal{O} \rho(\tau)]
$$

Let the observable $\mathcal{O}$ be an admissible atom state $\rho_{f}$, the probability $\mathscr{P}_{i \rightarrow f}$, that the atom evolves to the expected state represented by density matrix $\rho_{f}(\tau)$ from an initial one $\rho_{i} \equiv$ $\rho(0)$, should be

$$
\mathscr{P}_{i \rightarrow f}(\tau)=\operatorname{Tr}\left[\rho_{f} \rho(\tau)\right] .
$$

If initially the atom is in the ground state, its Bloch vector $|\rho(0)\rangle$ is $\{0,0,-1\}$, and the final state $\rho_{f}$ is the excited state given by the Bloch vector $\left|\rho_{f}\right\rangle=\{0,0,1\}$, according to (17)(22), we have

$$
\mathscr{P}_{i \rightarrow f}=\frac{1}{2}\left(1-e^{-4 A \tau}\right)\left(1-\frac{B}{A}\right) .
$$


The probability per unit time of the transition from the ground state to the excited state, in the limit of infinitely slow switching on and off the atom-field interaction, that is, the spontaneous excitation rate, can be calculated by taking the time derivative of $\mathscr{P}_{i \rightarrow f}(\tau)$ at $\tau=0$ as

$$
\Gamma_{i \rightarrow f}=\left.\frac{\partial}{\partial \tau} \mathscr{P}_{i \rightarrow f}(\tau)\right|_{\tau=0}=2 A-2 B=2 \mathscr{G}\left(-\omega_{0}\right)
$$

\section{Scalar Wave Equation Near the Event Horizon in a Kerr Space-Time}

3.1. Dimensional Reduction Near the Horizon. In order to find out how the reduced density evolves with proper time from (7), we will investigate the scalar wave equation of the Kerr space time. In Boyer-Lindquist coordinates, the stationary Kerr space time can be written as

$$
\begin{aligned}
d s^{2}= & -\frac{\Delta}{\rho^{2}}\left(d t-a \sin ^{2} \theta d \varphi\right)^{2} \\
& +\frac{\sin ^{2} \theta}{\rho^{2}}\left[\left(r^{2}+a^{2}\right) d \varphi-a d t\right]^{2} \\
& +\frac{\rho^{2}}{\Delta} d r^{2}+\rho^{2} d \theta^{2}
\end{aligned}
$$

where $\Delta=\left(r-r_{+}\right)\left(r-r_{-}\right), \rho^{2}=r^{2}+a^{2} \cos ^{2} \theta$ and $r_{ \pm}=$ $M \pm\left(M^{2}-a^{2}\right)^{1 / 2}$. The parameters $M$ and $a$ represent the mass and the angular momentum per unit mass of the black hole, respectively. The event horizon of the Kerr black hole is located at $r=r_{+}$. The line element in (25) is stationary and axisymmetric, with $\partial_{t}^{\mu}$ and $\partial_{\varphi}^{\mu}$ as the corresponding Killing vector fields.

And then, we will show that the scalar field theory in the background (25) can be reduced to a 2-dimensional field theory in the near-horizon region with the dimensional reduction technique. This technique firstly has been employed for the Kerr black hole by Murata and Soda [24] and developed with a more general technique by Iso et al. [23].

The action for the scalar field in a Kerr space time is

$$
S=\frac{1}{2} \int d x^{4} \sqrt{-g} g^{\mu \nu} \partial_{\mu} \phi \partial_{\nu} \phi+S_{\text {int }}
$$

where the first term is the kinetic term and the second term $S_{\text {int }}$ represents the mass, potential, and interaction terms.

By substituting (25) into (26), we obtain

$$
\begin{aligned}
S=-\frac{1}{2} \int d r d t d \theta d \varphi \sin \theta \phi & -\left(\frac{\left(r^{2}+a^{2}\right)^{2}}{\Delta}-a^{2} \sin ^{2} \theta\right) \partial_{t}^{2} \\
& -\frac{2 a\left(r^{2}+a^{2}-\Delta\right)}{\Delta} \partial_{t} \partial_{\varphi}
\end{aligned}
$$

$$
\begin{aligned}
& +\left(\frac{1}{\sin ^{2} \theta}-\frac{a^{2}}{\Delta}\right) \partial_{\varphi}^{2}+\partial_{r} \Delta \partial_{r} \\
& \left.+\frac{1}{\sin \theta} \partial_{\theta} \sin \theta \partial_{\theta}\right] \phi+S_{\text {int }}
\end{aligned}
$$

Now, we transform the radial coordinate $r$ into the tortoise coordinate $r_{*}$ defined by

$$
\frac{d r_{*}}{d r}=\frac{1}{F(r)} \equiv \frac{r^{2}+a^{2}}{\Delta}
$$

After the transformation, the action (27) can be written as

$$
\begin{aligned}
S=- & \frac{1}{2} \int d r_{*} d t d \theta d \varphi \sin \theta \phi \\
\times & -\left(\left(r^{2}+a^{2}\right)-F(r) a^{2} \sin ^{2} \theta\right) \partial_{t}^{2} \\
& \quad-2 a(1-F(r)) \partial_{t} \partial_{\varphi}+\left(\frac{F(r)}{\sin ^{2} \theta}-\frac{a^{2}}{r^{2}+a^{2}}\right) \partial_{\varphi}^{2} \\
& \left.+\partial_{r_{*}}\left(r^{2}+a^{2}\right) \partial_{r_{*}}+\frac{F(r)}{\sin \theta} \partial_{\theta} \sin \theta \partial_{\theta}\right] \phi+S_{\mathrm{int}}
\end{aligned}
$$

Now we consider this action in the region near the horizon. Since $F\left(r_{+}\right)=0$ at $r \rightarrow r_{+}$, we only retain dominant terms in (29). We have

$$
\begin{aligned}
S= & -\frac{1}{2} \int d r_{*} d t d \theta d \varphi \sin \theta \phi \\
& \times\left[-\left(r^{2}+a^{2}\right) \partial_{t}^{2}-2 a \partial_{t} \partial_{\varphi}\right. \\
& \left.-\frac{a^{2}}{r^{2}+a^{2}} \partial_{\varphi}^{2}+\partial_{r_{*}}\left(r^{2}+a^{2}\right) \partial_{r_{*}}\right] \phi
\end{aligned}
$$

where we have ignored $S_{\text {int }}$ by using $F\left(r_{+}\right)=0$ at $r \rightarrow$ $r_{+}$. Because the theory becomes high-energy case near the horizon and the kinetic term dominates, we can ignore all the terms in $S_{\text {int }}$. After this analysis, we return to the expression written in terms of $r$. So, we have

$$
\begin{aligned}
S=- & \frac{1}{2} \int d t d r d \theta d \varphi \sin \theta\left(r^{2}+a^{2}\right) \phi \\
\times & {\left[-\frac{1}{F(r)} \partial_{t}^{2}-\frac{2 a}{\Delta} \partial_{t} \partial_{\varphi}-\frac{a^{2}}{\Delta\left(r^{2}+a^{2}\right)} \partial_{\varphi}^{2}\right.} \\
& \left.+\partial_{r} F(r) \partial_{r}\right] \phi .
\end{aligned}
$$

Following Murata and Soda's method [24], we transform the coordinates to the corotating coordinate system. They 
employed a locally corotating coordinate system, and we will use a globally corotating coordinate system as

$$
\begin{gathered}
\psi=\varphi-\frac{a}{r^{2}+a^{2}} t, \\
\xi=t .
\end{gathered}
$$

Under these new coordinates, we can rewrite the action (31) as

$$
\begin{aligned}
S[\phi]= & -\frac{1}{2} \int d \xi d r d \psi d \theta\left(r^{2}+a^{2}\right) \\
& \times \sin \theta \phi\left(-\frac{1}{F(r)} \partial_{\xi}^{2}+\partial_{r} F(r) \partial_{r}\right) \phi,
\end{aligned}
$$

so the angular terms disappear completely. Using the spherical harmonics expansion $\phi=\Sigma_{l, m} \phi_{l m}(\xi, r) Y_{l m}(\theta, \psi)$, we obtain the effective 2 -dimensional action

$$
\begin{aligned}
S[\phi]= & \sum_{l, m} \frac{1}{2} \int\left(r^{2}+a^{2}\right) d \xi d r \phi_{l m} \\
& \times\left(-\frac{1}{F(r)} \partial_{\xi}^{2}+\partial_{r} F(r) \partial_{r}\right) \phi_{l m}
\end{aligned}
$$

where we have used the orthonormal condition for the spherical harmonics as follows:

$$
\int d \psi d \theta \sin \theta Y_{l^{\prime} m^{\prime}}^{*} Y_{l m}=\delta_{l^{\prime}, l} \delta_{m^{\prime}, m} .
$$

From the action (34), it is obvious to find that $\phi$ can be considered as a $(1+1)$-dimensional massless scalar field in the backgrounds of the dilaton $\Phi$. The effective 2-dimensional metric and the dilaton $\Phi$ can be written as

$$
\begin{gathered}
d s^{2}=-F(r) d \xi^{2}+\frac{1}{F(r)} d r^{2}, \\
\Phi=r^{2}+a^{2}
\end{gathered}
$$

So far, we have reduced the 4-dimensional field theory to a 2-dimensional case. This is consistent with [23]. This 2-dimensional metric tells us that, near the horizon, the geometry of a Kerr space time can be regarded as a Rindler space time when $r_{+}>r_{-}$. In the extremal case $r_{+}=r_{-}$, the near horizon geometry reduces to $A d S_{2}$ which is consistent with $[19,23]$. The same as the Schwarzschild space time [33], we will define two vacuum states by using the two natural notions of time translation of this effective 2-dimensional metric, namely, the Killing time and the proper time as measured by a congruence of freely falling observers.

3.2. The Boulware Vacuum. Using the tortoise coordinate in (28), the effective 2-dimensional metric (36) can be changed into

$$
d s_{I}^{2}=-F(r)\left(d \xi^{2}+d r_{*}^{2}\right) .
$$

We can see that the $\left(\xi, r_{*}\right)$ part of the metric has the form of Minkowski metric. Now in this 2-dimensional space time, the wave equation of $\phi\left(\xi, r_{*}\right)$ can be written as

$$
\left[\partial_{\xi}^{2}-\partial_{r_{*}}^{2}\right] \phi\left(\xi, r_{*}\right) \equiv \partial_{u} \partial_{v} \phi(u, v)=0
$$

Its standard ingoing and outgoing orthonormal mode solutions are

$$
\phi\left(\xi, r_{*}\right) \sim\left(e^{-i \sigma\left(\xi+r_{*}\right)}, e^{-i \sigma\left(\xi-r_{*}\right)}\right) \sim\left(e^{-i \sigma v}, e^{-i \sigma u}\right),
$$

where $v=\xi+r_{*}, u=\xi-r_{*}$ are null coordinates. These modes are positive frequency modes with respect to the killing vector field $\partial / \partial \xi$ for $\sigma>0$, and they satisfy

$$
L_{\partial / \partial \xi} \phi=-i \sigma \phi
$$

It is obvious that the wave equation (39) is manifestly invariant under the infinite-dimensional group of conformal transformation in two dimensions $u \rightarrow u^{\prime}(u), v \rightarrow v^{\prime}(v)$. In the following, we will show how this conformal symmetry does play the key role in the quantum effect of a Kerr space time.

Near the event horizon, we only consider the outgoing modes

$$
\phi^{\text {out }}\left(\xi, r_{*}\right)=\frac{1}{\sqrt{4 \pi \omega}} e^{-i \sigma\left(\xi-r_{*}\right)}=\frac{1}{\sqrt{4 \pi \omega}} e^{-i \sigma u},
$$

along the rays $u=$ constant. Quantizing the field $\phi^{\text {out }}$ in the exterior of the black hole, we can expand it as follows

$$
\widehat{\phi}^{I}=\sum_{\sigma}\left[a_{\sigma}^{I} \phi_{\sigma}^{\text {out }}\left(\xi, r_{*}\right)+a_{\sigma}^{I \dagger} \phi_{\sigma}^{\text {out } \dagger}\left(\xi, r_{*}\right)\right]
$$

where $a_{\sigma}^{I}$ and $a_{\sigma}^{I \dagger}$ are the annihilation and creation operators acting on the $I$ vacuum state, which corresponds to the Boulware vacuum. The Fock vacuum state can be defined as

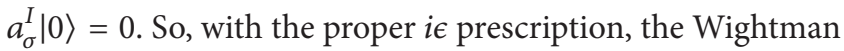
function of $I$ state can be written as

$$
G_{\text {Kerr }}^{I+}\left(x, x^{\prime}\right)=-\frac{1}{4 \pi^{2}} \frac{1}{\left(x^{0}-i \epsilon\right)^{2}-\left(x^{1}\right)^{2}}=-\frac{1}{4 \pi^{2}} \frac{1}{(\Delta \xi-i \epsilon)^{2}},
$$

where $x^{0}=\xi, x^{1}=r_{*}$.

3.3. The Unruh Vacuum. In order to define the Unruh vacuum state, one can write the Kerr line element in the nearhorizon region in terms of Kruskal-like coordinates defined as

$$
\begin{gathered}
U=T-R=-\kappa^{-1} e^{-\kappa u}, \\
V=T+R=\kappa^{-1} e^{\kappa \nu},
\end{gathered}
$$

where $T=\kappa^{-1} e^{\kappa r_{*}} \sinh \kappa \xi, R=\kappa^{-1} e^{\kappa r_{*}} \cosh \kappa \xi$ and $\kappa=\left(r_{+}-\right.$ $\left.r_{-}\right) / 2\left(r_{+}^{2}+a^{2}\right)$ is the surface gravity of the event horizon. The effective 2-dimensional space time (36) becomes

$$
d s_{I I}^{2}=C(r)\left[-d T^{2}+d R^{2}\right]
$$

where $C(r)=e^{-2 \kappa r_{*}} F(r)$, which is a finite constant near the event horizon $r=r_{+}$. The $(T, R)$ part of the metric also has the form of Minkowski metric. The interval of time $\Delta T$ then corresponds to the interval of proper time of a radial freely 
falling observer crossing the horizon. The 2-dimensional space time in the $(T, R)$ coordinates is well-behaved near the event horizon. One can obtain the wave equation of $\phi(T, R)$ as

$$
\left[\partial_{T}^{2}-\partial_{R}^{2}\right] \phi(T, R) \equiv \partial_{U} \partial_{V} \phi(U, V)=0
$$

Similar to previous proceeding, we can obtain the outgoing wave solution as $\phi^{\text {out }}(T, R) \sim e^{-i \omega(T-R)}=e^{-i \omega U}$. These modes are positive frequency modes with respect to the freely falling observer for $\omega>0$, satisfying

$$
L_{\partial / \partial_{T}} \phi=-i \omega \phi
$$

As pointed in previous subsection, there are conformal transformations from $\phi(U, V)$ to $\phi(u, v)$, as (45).

Subsequently, quantizing the field $\phi^{\text {out }}(T, R)$ in the exterior of the black hole, we can expand it as follows:

$$
\widehat{\phi}^{I I}=\sum_{\omega}\left[a_{\omega}^{I I} \phi_{\omega}^{\text {out }}(T, R)+a_{\omega}^{I I \dagger} \phi_{\omega}^{\text {out } \dagger}(T, R)\right]
$$

where $a_{\omega}^{I I}$ and $a_{\omega}^{I I \dagger}$ are the annihilation and creation operators acting on the $I I$ vacuum state, which can be defined as $a_{\omega}^{I I}|0\rangle=0$. This vacuum state is just the so-called Unruh vacuum defined in the maximally extended geometry. So, with the proper $i \epsilon$ prescription, the Wightman function of the II state can be written as

$$
\begin{aligned}
G_{\text {Kerr }}^{I I+}\left(x, x^{\prime}\right) & =-\frac{1}{4 \pi^{2}} \frac{1}{\left(x^{0}-i \epsilon\right)^{2}-\left(x^{1}\right)^{2}} \\
& =-\frac{1}{16 \pi^{2} \kappa^{-2} \sinh ^{2}\left[\left(\xi-\xi^{\prime}\right) \kappa / 2-i \epsilon\right]},
\end{aligned}
$$

where $x^{0}=T, x^{1}=R$.

\section{Probability of Spontaneous Transition of the Detector in a Kerr Space-Time}

In what follows, we will calculate the spontaneous excitation rate in the two vacuum states with the open quantum system approach.

4.1. The I State-Boulware Vacuum. Firstly, let us turn to the $I$ state-Boulware vacuum case. Thinking of the relation between the proper time and the coordinate time,

$$
d \tau=\sqrt{F(r)} d \xi
$$

the Fourier transform of the Wightman function (44) with respect to the proper time can be expressed as

$$
\begin{aligned}
\mathscr{G}_{\mathrm{Kerr}}(\lambda) & =\int_{-\infty}^{+\infty} d \tau e^{i \lambda \tau} G_{\mathrm{Kerr}}^{I+}\left(x, x^{\prime}\right) \\
& =-\int_{-\infty}^{+\infty} d \xi \sqrt{F(r)} e^{i \lambda \sqrt{F(r)} \xi}\left[\frac{1}{4 \pi^{2}} \frac{1}{(\Delta \xi-i \epsilon)^{2}}\right]=0 .
\end{aligned}
$$

According to (12), we have

$$
A=B=0 .
$$

So the spontaneous excitation rate can be obtained as

$$
\Gamma_{i \rightarrow f}=2(A-B)=0 .
$$

Therefore, no spontaneous excitation would ever occur in the $I$ state-Boulware vacuum. In fact, the Boulware vacuum corresponds to our familiar notion of a vacuum state. This result is consistent with the conclusion in [16].

4.2. The II State-Unruh Vacuum. Using the Wightman function (50) and the relation between the proper time and coordinate time (51), the Fourier transform can be given as

$$
\mathscr{G}_{\mathrm{Kerr}}(\lambda)=\int_{-\infty}^{+\infty} d \tau e^{i \lambda \tau} G_{\mathrm{Kerr}}^{I+}\left(x, x^{\prime}\right)=\frac{\lambda}{2 \pi} \frac{e^{2 \pi \kappa_{r}^{-1} \lambda}}{e^{2 \pi \kappa_{r}^{-1} \lambda}-1},
$$

where $\kappa_{r}=\kappa / \sqrt{F(r)}=\kappa \sqrt{\left(r^{2}+a^{2}\right) / \Delta}$.

According to (12), we have

$$
\begin{gathered}
A=\frac{1}{2}\left[\mathscr{G}_{\mathrm{Kerr}}\left(\omega_{0}\right)+\mathscr{G}_{\mathrm{Kerr}}\left(-\omega_{0}\right)\right]=\frac{\omega_{0} \operatorname{coth}\left(\pi \omega_{0} / \kappa_{r}\right)}{4 \pi}, \\
B=\frac{1}{2}\left[\mathscr{G}_{\mathrm{Kerr}}\left(\omega_{0}\right)-\mathscr{G}_{\mathrm{Kerr}}\left(-\omega_{0}\right)\right]=\frac{\omega_{0}}{4 \pi}, \\
C=\mathscr{G}_{\mathrm{Kerr}}(0)-\frac{\omega_{0} \operatorname{coth}\left(\pi \omega_{0} / \kappa_{r}\right)}{4 \pi} .
\end{gathered}
$$

Using (23) and (24), we have

$$
\begin{aligned}
\mathscr{P}_{i \rightarrow f}= & \frac{1}{2}\left(1-e^{-\left(\omega_{0} \operatorname{coth}\left(\pi \omega_{0} / \kappa_{r}\right) / \pi\right) t}\right) \\
& \times\left(1-\frac{1}{\operatorname{coth}\left(\pi \omega_{0} / \kappa_{r}\right)}\right),
\end{aligned}
$$

and the spontaneous excitation rate is

$$
\Gamma_{i \rightarrow f}=\left.\frac{\partial}{\partial t} \mathscr{P}_{i \rightarrow f}(t)\right|_{t=0}=\frac{\omega_{0}}{\pi\left(e^{2 \pi \omega_{0} / \kappa_{r}}-1\right)},
$$

which reveals that, the ground state detector in the $I I$ vacuum would spontaneously excite with an excitation rate that one would expect in the case of a flux of thermal radiation at the temperature

$$
T=\frac{\kappa_{r}}{2 \pi} \text {. }
$$

It is obvious that there is a near horizon conformal symmetry from $\phi(U, V)$ to $\phi(u, v)$ as (45), which is just the reason of the nonvanishing spontaneously excitation. This proposal is similar to [29]. In fact, the effective temperature $T$ in (59) approaches to Hawking temperature $T=\kappa / 2 \pi=\left(r_{+}-\right.$ $\left.r_{-}\right) / 4 \pi\left(r_{+}^{2}+a^{2}\right)$ as $r \rightarrow \infty$. This suggests that the thermal radiation emanating from the horizon of a Kerr black hole is just Hawking radiation, which is in agreement with $[16,17]$. 


\section{Conclusions and Discussions}

In brief, we have investigated the Hawking radiation from a Kerr space time through examining the evolution of a detector (modeled by a two-level atom) interacting with the vacuum massless scalar field in the framework of open quantum systems.

First of all, using the dimensional reduction technique, the 4-dimensional spherically nonsymmetric Kerr metric can be regarded as a 2-dimensional effective spherically symmetric metric near the event horizon. So we can construct two conformal vacuum states in this 2-dimensional effective space time: one is the $I$ state-Boulware vacuum, the other is the II state-Unruh vacuum. Then we give the Wightman functions of the two vacuum states, respectively.

On the basis of these, we have calculated the time evolution of the detector in the two vacuum states. It is found that the detector in the II state-Unruh vacuum would spontaneously excite with a nonvanishing probability the same as thermal radiation at Hawking temperature from a Kerr black hole. Hawking-Unruh effect of a Kerr space time can be understood as a manifestation of thermalization phenomena in an open quantum system. Meanwhile, it is also found that the probability of spontaneous transition of the detector would be vanishing in the I state-Boulware vacuum. It suggests that near horizon conformal symmetry plays the key role in the full quantum phenomena in the Kerr space time.

\section{Conflict of Interests}

The authors declare that there is no conflict of interests regarding the publication of this paper.

\section{Acknowledgments}

The authors would like to thank Dr. Kui Xiao and Dr. Shi-Wei Zhou for their helpful discussions and suggestions. This research is supported by the National Natural Science Foundation of China under Grant no. 11365008.

\section{References}

[1] S. W. Hawking, "Black hole explosions?" Nature, vol. 248, no. 5443, pp. 30-31, 1974.

[2] S. W. Hawking, "Particle creation by black holes," Communications in Mathematical Physics, vol. 43, no. 3, pp. 199-220, 1975.

[3] G. Gibbons and S. W. Hawking, "Action integrals and partition functions in quantum gravity," Physical Review D, vol. 15, pp. 2752-2756, 1977.

[4] T. Damour and R. Ruffini, "Black-hole evaporation in the KleinSauter-Heisenberg-Euler formalism," Physical Review D, vol. 14, no. 2, pp. 332-334, 1976

[5] S. Sannan, "Heuristic derivation of the probability distributions of particles emitted by a black hole," General Relativity and Gravitation, vol. 20, no. 3, pp. 239-246, 1988.

[6] M. K. Parikh and F. Wilczek, "Hawking radiation as tunneling," Physical Review Letters, vol. 85, no. 24, pp. 5042-5045, 2000.
[7] M. Parikh, "A secret tunnel through the horizon," International Journal of Modern Physics D, vol. 13, no. 10, pp. 2351-2354, 2004.

[8] M. K. Parikh, "Energy conservation andHawking radiation," http://arxiv.org/abs/hep-th/0402166.

[9] K. Xiao and W. B. Liu, "From Schwarzschild to Kerr under de Sitter background due to black hole tunnelling," Canadian Journal of Physics, vol. 85, no. 8, pp. 863-868, 2007.

[10] Q. Dai and W. B. Liu, "Hawking radiation from a spherically symmetric static black hole," Letters in Mathematical Physics, vol. 81, no. 2, pp. 151-159, 2007.

[11] W. Liu, "Massive tunneling in static black holes," Nuovo Cimento della Societa Italiana di Fisica B, vol. 122, no. 1, pp. 59-65, 2007.

[12] S. P. Robinson and F. Wilczek, "Relationship between Hawking radiation and gravitational anomalies," Physical Review Letters, vol. 95, no. 1, Article ID 011303, 4 pages, 2005.

[13] K. Xiao, W. Liu, and H. B. Zhang, "Anomalies of the AchucarroOrtiz black hole," Physics Letters B, vol. 647, no. 5-6, pp. 482-485, 2007.

[14] H.-P. Breuer and F. Petruccione, The Theory of Open Quantum Systems, Oxford University Press, Oxford, UK, 2002.

[15] F. Benatti and R. Floreanini, "Entanglement generation in uniformly accelerating atoms: reexamination of the Unruh effect," Physical Review A, vol. 70, no. 1, Article ID 012112, 12 pages, 2004.

[16] H. Yu and J. Zhang, "Understanding Hawking radiation in the framework of open quantum systems," Physical Review D, vol. 77, no. 2, Article ID 024031, 7 pages, 2008.

[17] H. Yu, "Open quantum system approach to the GibbonsHawking effect of de Sitter space-time," Physical Review Letters, vol. 106, no. 6, Article ID 061101, 4 pages, 2011.

[18] A. Strominger, "Black hole entropy from near-horizon microstates," Journal of High Energy Physics, vol. 1998, article 009, 1998.

[19] J. M. Bardeen and G. T. Horowitz, "Extreme Kerr throat geometry: a vacuum analog of $\mathrm{AdS}_{2} \times \mathrm{S}^{2}$," Physical Review D, vol. 60, no. 10, Article ID 104030, 10 pages, 1999.

[20] S. Carlip, "Black hole entropy from conformal field theory in any dimension," Physical Review Letters, vol. 82, no. 14, pp. 2828-2831, 1999.

[21] S. Carlip, "Black hole thermodynamics from Euclidean horizon constraints," Physical Review Letters, vol. 99, no. 2, Article ID 021301, 4 pages, 2007.

[22] S. N. Solodukhin, "Conformal description of horizon's states," Physics Letters B, vol. 454, no. 3-4, pp. 213-222, 1999.

[23] S. Iso, H. Umetsu, and F. Wilczek, "Anomalies, Hawking radiations, and regularity in rotating black holes," Physical Review D, vol. 74, no. 4, Article ID 044017, 10 pages, 2006.

[24] K. Murata and J. Soda, "Hawking radiation from rotating black holes and gravitational anomalies," Physical Review D, vol. 74, no. 4, Article ID 044018, 6 pages, 2006.

[25] M. Guica, T. Hartman, W. Song, and A. Strominger, "The Kerr/CFT correspondence," Physical Review D, vol. 80, no. 12, Article ID 124008, 9 pages, 2009.

[26] A. Castro and C. F. Larsen, "Near extremal Kerr entropy from $\mathrm{AdS}_{2}$ quantum gravity," Journal of High Energy Physics, vol. 2009, article 037, 2009.

[27] A. Castro, A. Maloney, and A. Strominger, "Hidden conformal symmetry of the Kerr black hole," Physical Review D, vol. 82, no. 2, Article ID 024008, 7 pages, 2010. 
[28] T. Hartman, W. Song, and A. Strominger, "Holographic derivation of Kerr-Newman scattering amplitudes for general charge and spin," Journal of High Energy Physics, vol. 2010, no. 3, article $118,2010$.

[29] I. Agullo, J. Navarro-Salas, G. J. Olmo, and L. Parker, "Hawking radiation by Kerr black holes and conformal symmetry," Physical Review Letters, vol. 105, no. 21, Article ID 211305, 4 pages, 2010.

[30] F. Benatti, R. Floreanini, and M. Piani, "Environment induced entanglement in Markovian dissipative dynamics," Physical Review Letters, vol. 91, no. 7, Article ID 070402, 4 pages, 2003.

[31] V. Gorini, A. Kossakowski, and E. C. G. Surdarshan, "Completely positive dynamical semigroups of $N$ level systems," Journal of Mathematical Physics, vol. 17, p. 821, 1976.

[32] G. Lindblad, "On the generators of quantum dynamical semigroups," Communications in Mathematical Physics, vol. 48, no. 2, pp. 119-130, 1976.

[33] N. D. Birrell and P. C. W. Davies, Quantum Fields in Curved Space, vol. 7, Cambridge University Press, Cambridge, UK, 1982. 

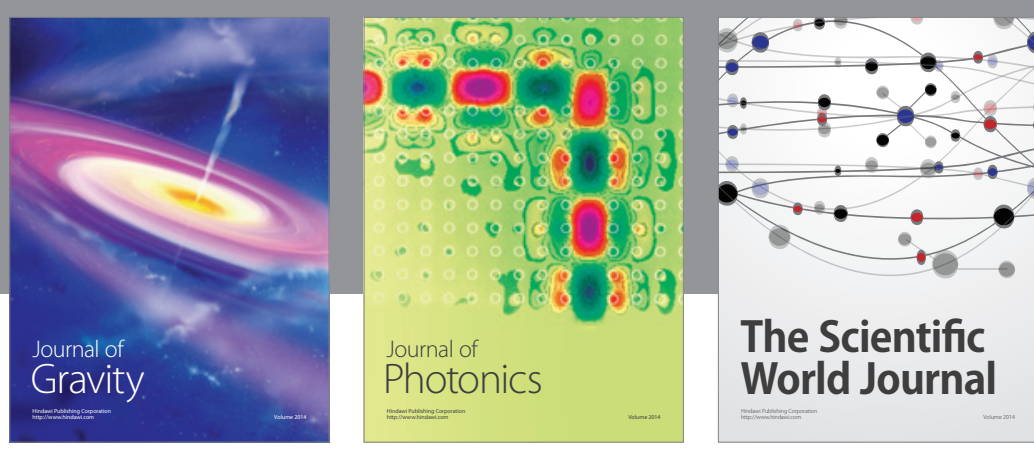

The Scientific World Journal
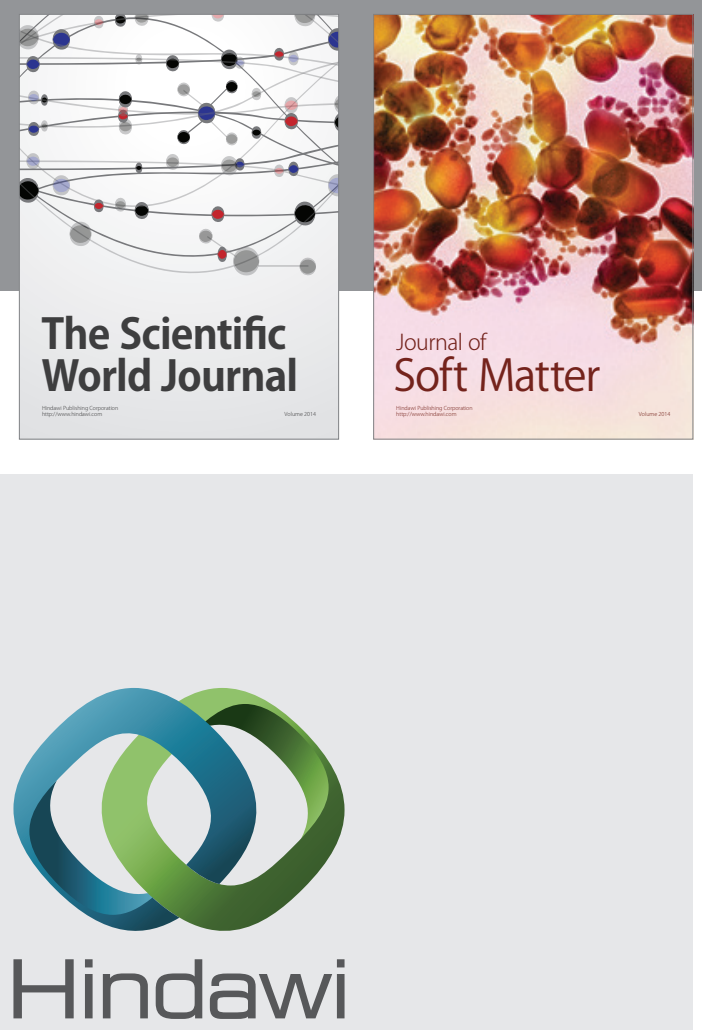

Submit your manuscripts at

http://www.hindawi.com

nternational Journal of

Statistical Mechanics
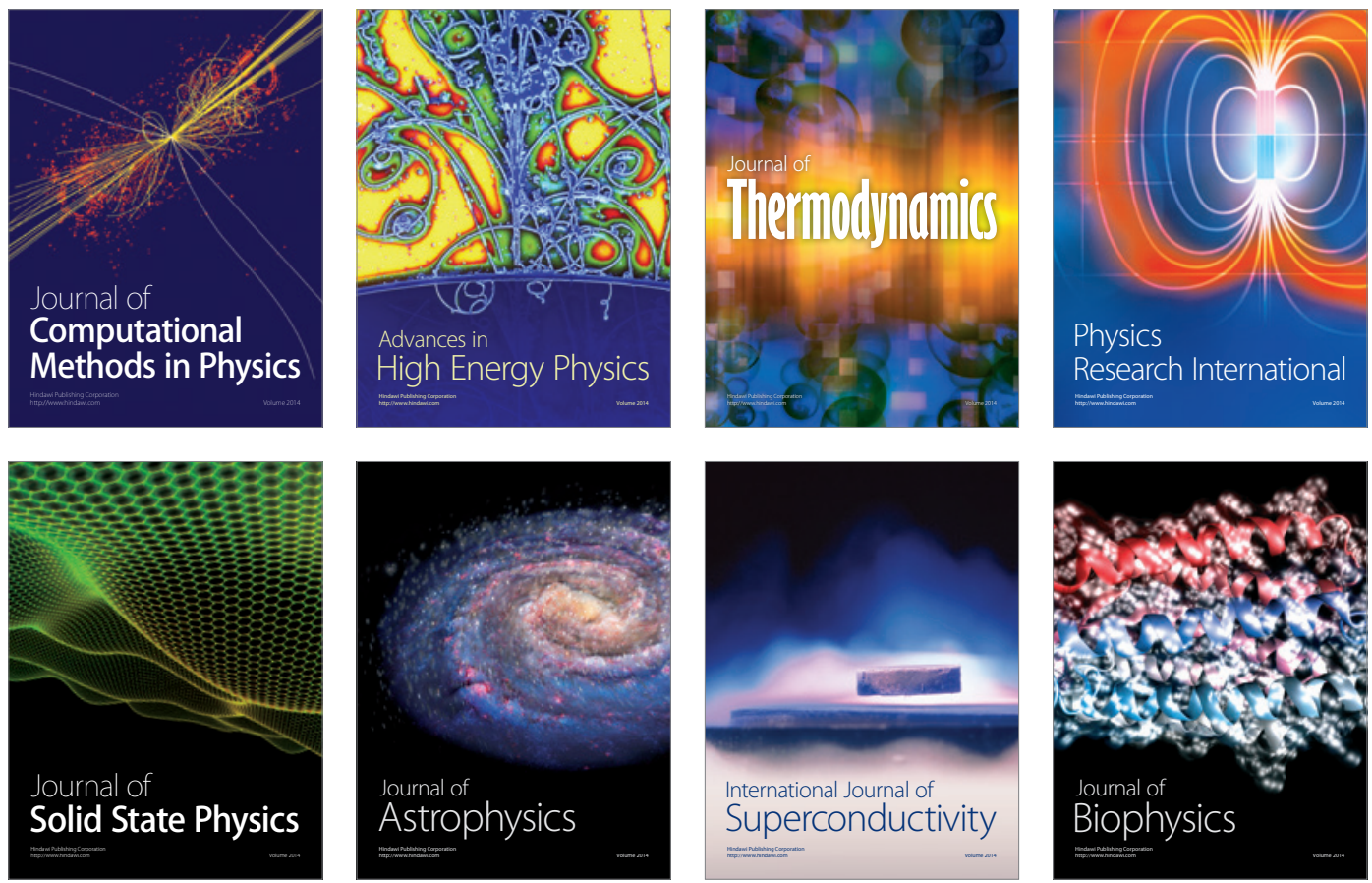
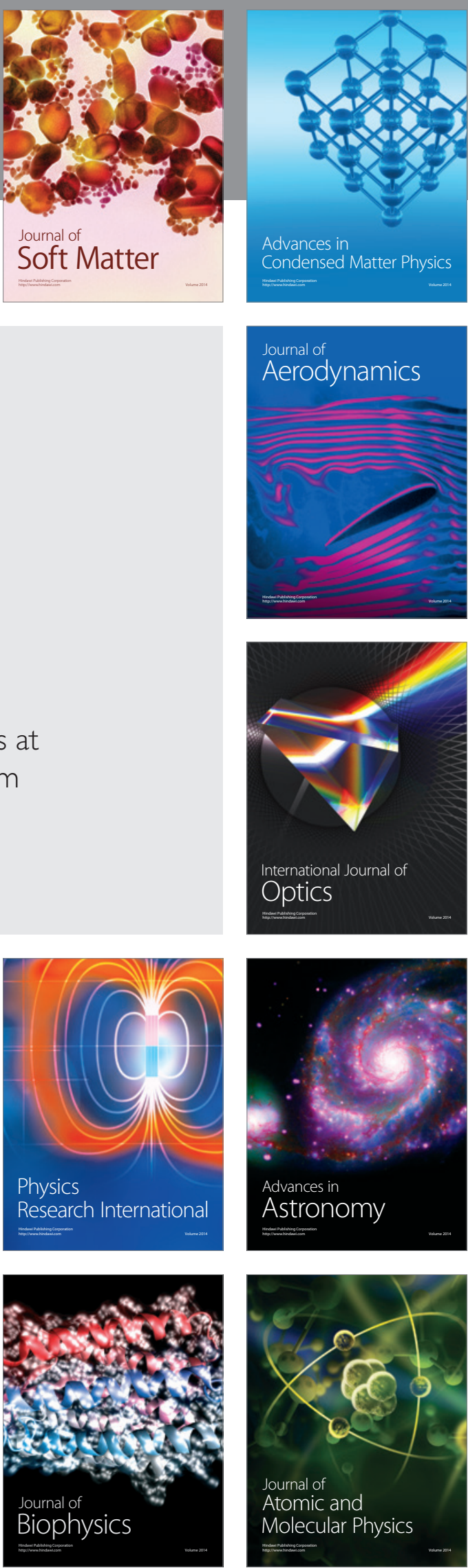